\title{
213 A-3
}

\section{Filter Design for Attitude Determination Using GPS and Gyro: Frequency Domain Approach}

\author{
Y. T. Chiang ${ }^{1}$, T. F. Wu ${ }^{1}$, F. R. Chang ${ }^{1}$ and L. S. Wang ${ }^{2}$ \\ 1 Department of Electrical Engineering, National Taiwan University, Taipei, Taiwan, R. O. C. \\ 2 Institute of Applied Mechanics, National Taiwan University, Taipei, Taiwan, R. O. C. \\ frchang@ac.ee.ntu.edu.tw
}

\begin{abstract}
A constant gain estimator is proposed to integrate the GPS and gyro measurements for attitude determination. Through frequency domain approach, a pair of high pass and low pass filters are employed. Concerning GPS and gyros errors, we apply Power Spectral Density (PSD) properties to select the cut-off frequencies. Once these values are determined, we can derive the associated Kalman gains. Since the PSD specifications of gyros are provided by equipment manufactory, the Kalman gains can be designed in advance: The reduction of the burdens of on-line computations, which are unavoidable in the traditional Kalman, make our algorithms suitable for real systems. From the assessment of software simulations, the proposed filter is sound and effective
\end{abstract}

Keywords: Kalman gain, Global Positioning System (GPS), Power Spectral Density (PSD)

\section{Introduction}

The attitude of a flight vehicle is its orientation in the air. A great deal of aircraft operations heavily depend on the attitude. A constant covariance estimator is proposed to integrate the GPS and gyro measurements. The unity norm quaternion representation, which does not cause singularity difficulty, is adopted to describe the attitude. 'The attitude error is defined as the rotation of the body frame from estimated values to true values. The aim of attitude filter is to minimize errors in the estimation.

The GPS satellites will broadcast their ephemeries and the time, which allow GPS receiver to determine its own position from simultaneous observations of any four or more GPS satellites. Furthermore, if three or more GPS receivers are installed on the edges of an aircraft, the pitch, roll and yaw angle of the platform can be computed by interfernometric processing of carrier phase observables. The data rate is $1 \mathrm{~Hz}$. On the other hand, gyro measurements supply the angular velocities directly at a higher data rate of 10 to $20 \mathrm{~Hz}$. To combine these measurements in different rates, an integrated architecture is proposed. The attitude propagation operation is based on the gyro measurements after bias error correction. The attitude updated operation is depend on the GPS observables.

Assuming that the angular velocity of the vehicle is constant, the steady state error covariance of Kalman filter can be derived. Through the frequency domain approach, we can develop an appropriate transfer function to acquire the optimal attitude. According to the noise characteristics of GPS and gyros measurements, the estimator can eliminate the low frequency gyro noise and the high frequency GPS noise, respectively.

To check our algorithms, software simulations are conducted. In scenarios of simulations include a twodegree-of-freedom rotational platform with two aluminum bars. Four GPS antennae is mounted at the ends of the two bars to form a non-colinear frame, and three rate gyros are installed on the platform to obtain the angular velocity. According to the results of the simulations, the proposed scheme is sound and effective.

\section{Models and Kinematics}

\subsection{GPS and Gyro Models}

After compensating the ambiguity values, the double difference carrier phase observables become ambiguity-free. The ambiguity-free double difference, $\psi_{i}^{j}$, is the inner product of the $i$-th baseline vector and the difference of the directional vectors to two satellites (the $j+1)$ th and the 1st). Let $\bar{q}$ be the quaternion representation of the body frame relation to the reference frame. The attitude matrix $A(\overline{\mathbf{q}})$ transforms the coordinates of vectors in the reference frame to those in the body frame. Accordingly, considering measurement noises, we have

$$
\begin{aligned}
\psi_{i}^{j} & =\mathbf{a}_{i}^{T} A(\overline{\mathbf{q}}) \mathbf{s}^{j}+\omega_{i}^{j} \\
& =\mathbf{r}_{i}^{T} \mathbf{s}^{j}+\omega_{i}^{j},
\end{aligned}
$$


where the vectors $\mathbf{a}_{i}$ and $\mathbf{r}_{i}$ represent the $i$-th baseline vector in the body coordinate and in the reference frame, respectively; $\mathrm{s}^{j}$ equals the difference between directional vector satellite $j+1$ and satelite 1 repre sented in the reference coordinate system; $\psi_{i}^{j}$ is the corresponding ambiguity-free double-difference phase measurements; the error vector $\omega_{i}^{j}$ is assumed to be white with distribution $\mathbb{N}(0.0 .01 \lambda)$.

The gyro output vector $\mathbf{u}$ is related to the true angular velocity of the body frame, $\omega$ by [1]

$$
\mathbf{u}=\omega+\mathbf{b}+\eta_{1}
$$

where $\mathbf{b}\left(=\mathbf{b}_{0}+\mathbf{b}_{w}\right)$ is the gyro drift bias vector, in which $\mathbf{b}_{0}$ denotes a constant bias and $\mathbf{b}_{w}$ denotes a random walk, and $\eta_{1}$ represents zero mean white Gaussian noise with strength $Q_{1}$. The drift bias is assumed to satisfy

$$
\frac{d}{d t} \mathbf{b}=\eta_{2}
$$

where $\eta_{2}$ is another zero mean white Gaussian noise with strength $Q_{2}$. The estimation of $\omega$ is formed by taking expectation of (2).

$$
\hat{\omega}=u-\hat{b}
$$

where $\hat{\mathbf{b}}$ is the estimation of $\mathbf{b}$. In the later development, the gyro drift bias $b$.

\subsection{Attitude Kinematics}

Let $\mathbf{A}$ be an attitude matrix, represented by the quaternion $\overline{\mathbf{q}}$ defined as

$$
\overline{\mathbf{q}}=\left[\begin{array}{c}
q_{1} \\
q_{2} \\
q_{3} \\
q_{4}
\end{array}\right]=\left[\left[\begin{array}{c}
\mathrm{L} \sin (\theta / 2) \\
\cos (\theta / 2)
\end{array}\right]\right]=\left[\begin{array}{c}
\mathrm{q} \\
q_{4}
\end{array}\right] .
$$

where $\mathbf{L}$ is a unit vector represents the axis of rotation; $\theta$ is the angle of rotation about the axis $L$. It is obvious that the norm of $q$ and $\bar{q}$ are $\left|\sin \left(\frac{\theta}{2}\right)\right|$ and 1 , respectively.

According to the relation

$$
\mathbf{A}(\overline{\mathbf{q}})=\left(\left|q_{4}\right|^{2}-|\mathbf{q}|^{2}\right) \mathbf{I}+2 \mathbf{q} \mathbf{q}^{T}+2 q_{4}[|\mathbf{q}|]
$$

where $\mathbf{I}$ is the $3 \times 3$ identity matrix and

$$
\begin{gathered}
{\left[\mid \mathbf{q} \|=\left[\begin{array}{ccc}
0 & q_{3} & -q_{2} \\
-q_{3} & 0 & q_{1} \\
q_{2} & -q_{1} & 0
\end{array}\right]\right.} \\
\overline{\mathbf{q}}^{T} \overline{\mathbf{q}}=1 .
\end{gathered}
$$

The kinematic relation between the attitude quaternion and the angular velocity is

$$
\frac{d}{d t} \overline{\mathbf{q}}(t)=\frac{1}{2} \Omega(\omega(t)) \overline{\mathbf{q}}(t)
$$

where

$$
\boldsymbol{\Omega}(\omega)=\left[\begin{array}{cccc}
0 & \omega_{3} & -\omega_{2} & \omega_{1} \\
-\omega_{3} & 0 & \omega_{1} & \omega_{2} \\
\omega_{2} & -\omega_{1} & 0 & \omega_{3} \\
-\omega_{1} & -\omega_{2} & -\omega_{3} & 0
\end{array}\right]
$$

and $\omega=\left[\begin{array}{lll}\omega_{1} & \omega_{2} & \omega_{3}\end{array}\right]$ denotes the angular velocity of the body.

To obtain the discretized model, we consider the time interval $t_{k}$ to $t_{k+1}$. In such a short period, $\hat{\omega}=$ $\mathbf{u}(t)-\hat{\mathbf{b}}(t)$ can be taken as a constant. Therefore, the expectation of equation (9) can be integrated directly from $t_{k}$ to $t_{k+1}$ :

$$
\hat{\overline{\mathbf{q}}}_{k+1}=\Theta_{k} \hat{\overline{\mathbf{q}}}_{k}
$$

where

$$
\Theta_{k}=\exp \left(\frac{1}{2} \int_{t_{k}}^{t_{k+1}} \Omega(\mathbf{u}-\hat{\mathbf{b}}) d \tau\right)
$$

\section{Linearized Kalman Filter}

\subsection{Dynamic Equations}

In stead of the $\overline{\mathbf{q}}(t)$ itself, the aim of our filter is to track its errors. Let define

$$
[\Delta \overline{\mathbf{q}}]=\left[\overline{\mathbf{q}} \hat{\mathbf{q}}^{-1}\right],
$$

where $\Delta \bar{q}$ is the quaternion error between the true attitude and the estimated attitude.

The advantage of the quaternion error representation is that the fourth component will be close to unity since the incremental quaternion corresponding to a small angle rotation. Thus the attitude information of interest is contained in the three tuple vector $\Delta \mathbf{q}$, where

$$
\Delta \overline{\mathbf{q}}=\left[\begin{array}{c}
\Delta \mathbf{q} \\
\sqrt{1-|\Delta \mathbf{q}|}
\end{array}\right] \cong\left[\begin{array}{c}
\Delta \mathbf{q} \\
1
\end{array}\right]=\left[\begin{array}{c}
\frac{1}{2} \theta_{x} \\
\frac{1}{2} \theta_{y} \\
\frac{1}{2} \theta_{z} \\
1
\end{array}\right]
$$

The reduced representation of state error vector can be defined by

$$
\mathbf{x}=\left[\begin{array}{l}
\theta \\
\mathbf{d}
\end{array}\right] .
$$

where $\mathbf{d}=\hat{\mathbf{b}}-\mathbf{b}$ is the drift bias error. The dynamic equations associated to the reduced state error representation will be

$$
\frac{d}{d t} \mathbf{x}(t)=\mathbf{F} \mathbf{x}(t)+\mathbf{G w}(t)
$$


where

$$
\mathbf{F} \equiv\left[\begin{array}{cc}
\|\mathbf{u}-\hat{\mathbf{b}}\| & \mathbf{I}_{3 \times 3} \\
\mathbf{0}_{3 \times 3} & \mathbf{0}_{3 \times 3}
\end{array}\right], \mathbf{G} \equiv\left[\begin{array}{cc}
-\mathbf{I}_{3 \times 3} & \mathbf{0}_{3 \times 3} \\
\mathbf{0}_{3 \times 3} & \mathbf{I}_{3 \times 3}
\end{array}\right],
$$

and $\mathbf{w}(t)=\left[\begin{array}{ll}\eta_{1}(t) & -\eta_{2}(t)\end{array}\right]^{T}$.

\subsection{Measurement Equations}

To avoid being overwhelmed by complex notations, we consider the case of one baseline vector first. The coordinates of baseline vectors represented in the body frame and in the reference frame can be related by the quaternion expression $\overline{\mathbf{q}}$ as

$$
\left[\begin{array}{c}
a_{x} \\
a_{y} \\
a_{z} \\
0
\end{array}\right]=\overline{\mathbf{q}} \otimes\left[\begin{array}{c}
r_{1} \\
r_{2} \\
r_{3} \\
0
\end{array}\right] \otimes \overline{\mathbf{q}}^{*}
$$

where $\left[\begin{array}{lll}a_{x} & a_{y} & a_{z}\end{array}\right]^{T}$ denotes the baseline vectors in the body frame and [ $\left.\begin{array}{lll}r_{1} & r_{2} & r_{3}\end{array}\right]^{T}$ denotes the baseline vectors in the reference frame. Based on (18), the measurement equation can be shown as

$$
\left[\begin{array}{l}
\hat{a}_{x}-a_{x} \\
\hat{a}_{y}-a_{y} \\
\hat{a}_{z}-a_{z}
\end{array}\right]=\left[\begin{array}{ll}
\|\mathbf{a}\| & \mathbf{0}_{3 \times 3}
\end{array}\right][\mathbf{x}]
$$

where $\left[\begin{array}{lll}\hat{a}_{x} & \hat{a}_{y} & \hat{a}_{z}\end{array}\right]^{T}$ is the predicted baseline vectors.

Concerning $m$, baseline vectors, the measurement equations can be expressed as

$$
\mathbf{z}=\mathbf{H} \mathbf{x}+\mathbf{v},
$$

where $\mathbf{x}$ id defined in (15)

$$
\begin{gathered}
\mathbf{z}=\left[\begin{array}{c}
\mathbf{z}_{1} \\
\mathbf{z}_{2} \\
\vdots \\
\mathbf{z}_{m}
\end{array}\right], \mathbf{z}_{i}=\left[\hat{\mathbf{a}}_{i}-\mathbf{a}_{i}\right], \\
\mathbf{H}=\left[\begin{array}{c}
\mathbf{h}_{1} \\
\mathbf{h}_{2} \\
\vdots \\
\mathbf{h}_{m}
\end{array}\right], \mathbf{h}_{i}=\left[\begin{array}{ll}
\left\|\mathbf{a}_{i}\right\| & \mathbf{0}_{3 \times 3}
\end{array}\right],
\end{gathered}
$$

the subscript $i$ represents the $i$-th baseline. The measured noise, $\mathbf{v}$, under assumption for simplicity, is assumed a zero mean white noise with covariance matrix $\mathbf{R}=\sigma^{2} \mathbf{I}_{3 m}$.

\section{Simplifications by Frequency Domain Approach}

The constant Kalman gain will assume the optimal estimation performance in steady state. In such a case, the computation load of Kalman filter is greatly reduced.

\subsection{Diagonal Forms of $\mathrm{KH}$}

Since the state $\mathbf{x}$ is a 6 tuple vector as known in (15), the covariance matrix of the estimation error is

$$
\mathbf{P}=\left[\begin{array}{ll}
\mathbf{P}_{1} & \mathbf{P}_{3} \\
\mathbf{P}_{2} & \mathbf{P}_{4}
\end{array}\right] \text {, a } 6 \times 6 \text { matrix }
$$

where $\mathbf{P}_{1}, \mathbf{P}_{2}, \mathbf{P}_{3}, \mathbf{P}_{4}$ are $3 \times 3$ matrices. The observation matrix $\mathbf{H}$ can be written as $\mathbf{H}=\left[\begin{array}{ll}\mathbf{H}_{\mathrm{I}} & \mathbf{0}_{3 m \times 3}\end{array}\right]$, where $\mathbf{H}_{\mathbf{I}}$ is a $3 m \times 3$ matrix (for $m$ baselines, and $m=3$ ). Then the computed $\mathbf{K}$ yields

$$
\mathbf{K}=\left[\begin{array}{c}
\mathbf{P}_{1} \mathbf{H}_{\mathbf{I}}^{T}\left(\mathbf{H}_{\mathbf{I}} \mathbf{P}_{\mathbf{1}} \mathbf{H}_{\mathbf{I}}^{T}+\mathbf{R}\right)^{-1} \\
\mathbf{P}_{2} \mathbf{H}_{\mathbf{I}}^{T}\left(\mathbf{H}_{\mathbf{I}} \mathbf{P}_{\mathbf{1}} \mathbf{H}_{\mathbf{I}}^{T}+\mathbf{R}\right)^{-1}
\end{array}\right],(6 \times 3 m),
$$

where $\mathbf{R}$ is the covariance matrix of measurement noises. Note that $\mathbf{P}_{3}, \mathbf{P}_{4}$ do not appear in the expression of $\mathbf{K}$. The associated expressed of $\mathbf{K H}$ can be expressed as

$$
\mathbf{K H}=\left[\begin{array}{cc}
(\mathbf{K H})_{\text {attitude }} & \mathbf{0}_{3 \times 3} \\
(\mathbf{K H})_{\text {drift }} & \mathbf{0}_{3 \times 3}
\end{array}\right] .
$$

The infinitesimal angle assumption is appropriate since we are dealing with the error of quaternions. The 3 small rotational angles, which represent the true attitude and the estimated attitude, can be considered as independent random variables with the same variance. Hence, $\mathbf{P}_{1}=P_{a} \mathbf{I}_{3}$. The covariance of gyro drift bias errors drift bias is assumed to be $\mathbf{P}_{2}=P_{d} \mathbf{I}_{3}$ for the similar consideration. Then we have $(\mathbf{K H})_{\text {attitude }}=l_{a} \mathbf{I}_{3}$, where $l_{a}=\frac{2 P_{a}}{2 P_{a}+\sigma_{a}^{2}}$, and $(\mathbf{K H})_{d r i f t}=l_{d} \mathbf{I}_{3}$, where $l_{d}=\frac{2 P_{d}}{2 P_{a}+\sigma_{a}^{2}}$, respectively

\subsection{Transfer Functions in Terms of GPS and Gyro}

The frequency domain model can simplify the 6 order state equations to 3 second order transfer functions.

$$
\begin{gathered}
\hat{o}=\frac{l_{a} \cdot s+l_{d}}{s^{2}+l_{a} \cdot s+l_{d}} \theta_{G P S}+\frac{s^{2}}{s^{2}+l_{a} \cdot s+l_{d}} \theta_{g y r o} \\
\hat{d}=\frac{l_{d} \cdot s}{s^{2}+l_{a} \cdot s+l_{d}}\left(\theta_{G P S}-\theta_{g y r o}\right)
\end{gathered}
$$

where $\theta_{C P S}$ is the measured attitude which is obtained from GPS, $\theta_{\text {gyro }}$ is the predicted attitude which is obtained by gyros, $l_{a}$ and $l_{d}$, respectively, are the updating 


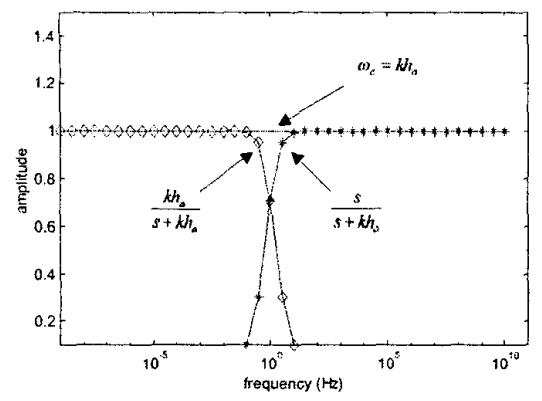

Figure 1: Frequency representation of the attitude estimation filter

gain of attitude and drift on the considered axis. The convergence time of the estimation filter can be related to the gains $l_{a}$ and $l_{d}$ by considering the poles of the above dynamic equation. Assuming $l_{a}>>l_{d}$ we have

$$
s^{2}+l_{a} s+l_{d} \approx\left(s+l_{a}\right)\left(s+\frac{l_{d}}{l_{a}}\right) .
$$

Therefore, setting

$$
T_{a b}=\frac{1}{l_{a}} \text { and } T_{d}=\frac{l_{a}}{l_{d}}
$$

$T_{a}$ and $T_{d}$ are homogeneous to convergence time of the estimation filter, respectively for attitude and for drift.

\subsection{Mono-Axis Analysis}

The attitude only estimation filter can be obtained from (26) while ignoring the drift updating gain $l_{d}$ :

$$
\hat{\theta}=\frac{l_{a}}{s+l_{a}} \theta_{G P S}+\frac{s}{s+l_{a}} \theta_{g y r o} .
$$

The attitude estimation error, based on (30), can be assumed as

$$
\widetilde{\theta}=\frac{l_{a}}{s+l_{a}} \epsilon_{G P S}+\frac{s}{s+l_{a}} \epsilon_{g y r o}=\tilde{\theta}_{C P S}+\widetilde{\theta}_{g y r o},
$$

where $\widetilde{\theta}=\hat{\theta}-\theta$ is the attitude estimation error, $\epsilon_{G P S}$ is the GPS measurement error; $\epsilon_{g y r o}$ is the gyros measurement error on attitude and $l_{a}$ is the Kalman filter gain on attitude. This expression comprise two filters with the same cut-off pulsation $l_{a}$ : a low pass filter which filters the high frequency GPS noise and a high pass filter which filters the low frequency gyros noise. The frequency representation of the attitude estimation filter is shown in Figure 1. In this figure, $\omega_{c}$ is the identical pulsation among which the high pass filter and the low pass filter. The tuning process for attitude estimation is to select a optimal $l_{a}$ :

$$
T_{a}=\frac{1}{l_{a}}=\frac{1}{\omega_{c}},
$$

where $\omega_{c}$ is in rad/sec.

\section{$5 \quad$ PSD and Kalman Gain}

\subsection{PSD representations for Parameter selecting}

The estimate attitude error expressed in (31) consists of GPS estimate attitude error and grro estimate attitude error, through use of a high pass filter and a low pass filter which filter the GPS and gyro noises, respectively. While assuming the two attitude errors is uncorrelated, the PSD of the attitude error can be expressed as

$$
P S D(\widetilde{\theta})=\frac{l_{a}^{2}}{l_{a}^{2}+\omega^{2}} P S D_{G P S}+\frac{\omega^{2}}{l_{a}^{2}+\omega^{2}} P S D_{g y r o} .
$$

To minimize the PSD of the attitude error through choose a optimal $l_{a}$, we differentiate (??) with respect. to $l_{a}$ :

$$
\frac{d}{d\left(l_{a}\right)} P S D(\widetilde{\theta})=\frac{2\left(l_{a}\right) \cdot \omega^{2}}{\left(l_{a}^{2}+\omega^{2}\right)^{2}}\left(P S D_{G P S}-P S D_{g y r o}\right) .
$$

Equation (34) shown that the optimal selected parameter of the $l_{a}$ is chosen as $P S D_{G P S}$ equal to $P S D_{\text {gyro. }}$.

\subsection{GPS and Gyro Noises}

Given a phase measured error of $\sigma_{p}$, applying relative positioning, the expected baseline error is calculated using the following expression

$$
\sigma_{a}=R G D O P \cdot \sigma_{p},
$$

where $R G D O P$ which is described in [4], $R G D O P=$ $\sqrt{2} P D O P$.

The resulting total angular pointing error $\sigma_{A}$ may be written as follows:

$$
\sigma_{A}=\frac{\sigma_{a}}{L_{E}}
$$

where $L_{E}$ is the effective baseline length. From the description above, the attitude noise caused by GPS carrier phase observables is assumed equivalent to a white noise which PSD is computed as

$$
P S D_{C P S}=\frac{2 \sigma_{A}^{2}}{f_{C P S}}=\frac{4 P D O P \cdot \sigma_{p}^{2}}{f_{C P S} \cdot L_{E}}
$$


where $f_{C P S}$ is the data rate of GPS observables .

The stochastic model of gyro noise is described in [2]. According to the model, the PSD of gyro noises is computed as

$$
P S D_{g y r o}(\omega)=\frac{2 K_{n}^{2}}{\omega^{4}}+\frac{2 B_{n}^{2}}{\omega^{3}}+\frac{2 N_{n}^{2}}{\omega^{2}}+2 \Phi_{n}^{2} .
$$

\subsection{Selection of Kalman Gain $K$}

In this Simulations, the noise standard deviation of GPS carrier phase observables is chosen as $0.01 \lambda=$ $0.19 \mathrm{~cm}$ and baseline length is $1 \mathrm{~m}$. Assuming $P D O P=$ 1, from (37), we have $P S D_{C P S}=1.44 \times 10^{7} \mathrm{\mu rad}^{2} / \mathrm{Hz}_{\mathrm{z}}$.

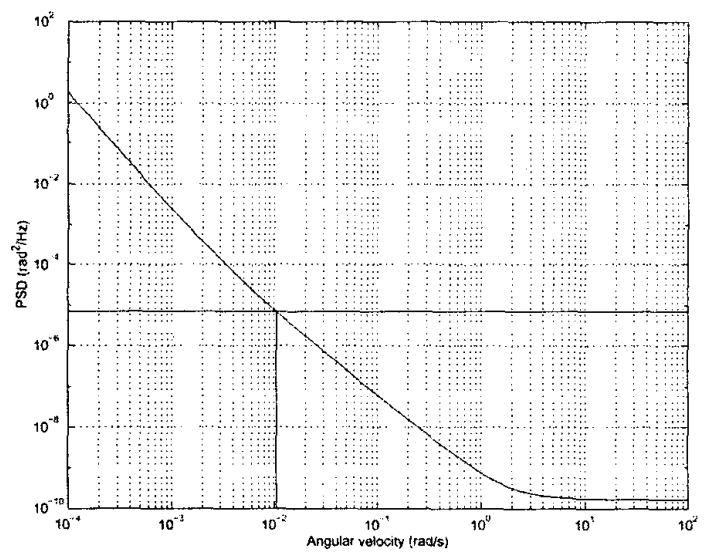

Figure 2: Preliminary tuning of GPS-gyro filter using PSD representation

The gyros noise specifications, according to [2], are assumed to be $B_{n}=0.2 \mathrm{deg} / \mathrm{hr}, N_{n}=$ $0.06 \mathrm{deg} / \operatorname{sqrt}(h r), \quad \Phi_{n}=1.9 \% / \operatorname{sqrt}(H z), \quad K_{n}=$ $0 \mathrm{deg} / \mathrm{h} \mathrm{r}^{3 / 2}$.

From Figure 2, the estimation parameter of attitude convergence time can be chosen as

$$
T_{a}=\frac{1}{\omega_{c}}=\frac{1}{l_{a}}=100 \mathrm{~s} .
$$

According to the descriptions of $P_{a}$ and $l_{a}$ in section 4.1, we have

$$
P_{a}=\frac{l_{a}}{2\left(1-l_{a}\right)} \sigma_{A}^{2}=1.823 \times 10^{4} \mu \mathrm{rad} d^{2} .
$$

Assuming that The convergence time of the drift estimation process is selected to $T_{d}=200 \mathrm{~s}$, we have $P_{d}=\frac{P_{0}}{T_{d}}=91.15 \mu \mathrm{rad}^{2}$. Then the computation of the $\mathrm{K}$, expressed in (24), yields

$$
\mathbf{K}=\left[\begin{array}{l}
\mathbf{P}_{1} \mathbf{H}_{\mathrm{I}}^{T}\left(\mathbf{H}_{\mathbf{I}} \mathbf{P}_{1} \mathbf{H}_{\mathbf{I}}^{T}+\mathbf{R}\right)^{-1} \\
\mathbf{P}_{2} \mathbf{H}_{\mathbf{I}}^{T}\left(\mathbf{H}_{\mathbf{I}} \mathbf{P}_{1} \mathbf{H}_{\mathbf{I}}^{T}+\mathbf{R}\right)^{-1}
\end{array}\right],
$$

where $\mathbf{P}_{1}=P_{4} \mathbf{I}_{3}$ and $\mathbf{P}_{2}=P_{d} \mathbf{I}_{3}$.

\section{Filter Operations and Simula- tions}

\subsection{Operations of GPS-Gyro Filter}

The filter design algorithms for attitude determination is built up in Figure 3. The system propagation operation is based on the gyro measurements with $16 \mathrm{~Hz}$ lata rate. The estimate of state vector, which is used to correct the attitude quaternion, is updated by GPS measurements with $1 \mathrm{~Hz}$ data rate.

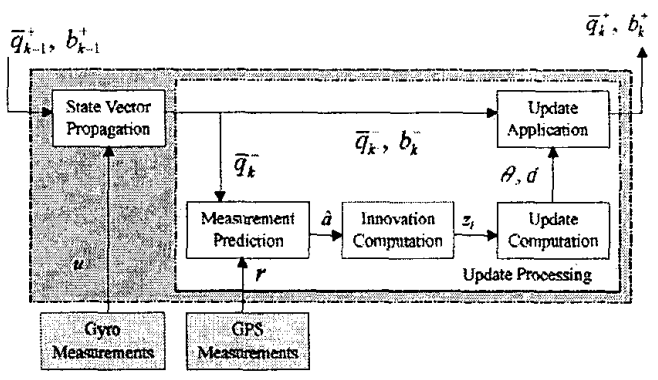

Figure 3: Architecture diagram of GPS-Gyro filter

\subsection{Simulations}

To verify the filter algorithm for attitude determination, a pratical simulation is used to assess the performance of our method. The scenario of the simulations are assumed to be

(1) There are 3 orthogonal baselines which are coincided with the 3 axes of body frame are chosen. In other word, they are $\mathbf{a}_{1}=\left[\begin{array}{ccc}1 & 0 & 0\end{array}\right]^{T}, \mathbf{a}_{2}=\left[\begin{array}{lll}0 & 1 & 0\end{array}\right]^{T}$ and $\mathbf{a}_{3}=\left[\begin{array}{lll}0 & 0 & 1\end{array}\right]^{T}$, respectively.

(1) The angular velocities: $\omega=\left[\begin{array}{lll}0 & 0 & 1.0 \times 10^{-3}\end{array}\right]^{T} \mathrm{rad} / \mathrm{sec}$.

(2) Gyro output data: - data rate: $16 \mathrm{~Hz}$. - constant drift error: $30 \mathrm{deg} / \mathrm{hr}$. .

- constant scale factor error: $750 \mathrm{ppm}$.

(3)GPS output data: - data rate: $1 \mathrm{~Hz}$. - noise $(1 \sigma)$ : $0.11 \mathrm{deg}$ on $\mathrm{roll} / \mathrm{pitch} /$ yaw axes.

Figure 4 shows the estimations of attitude errors with initial constant drift biases. The main part of the attitude errors are invoked by gyro measurements due to the time need for filter convergence on drift biases estimation. The estimated gyro drift biases are shown 
in Figure 5. After calibrating drift bias, the attitude can be accurate estimated. The attitude errors in steady state are shown in Figure 6.
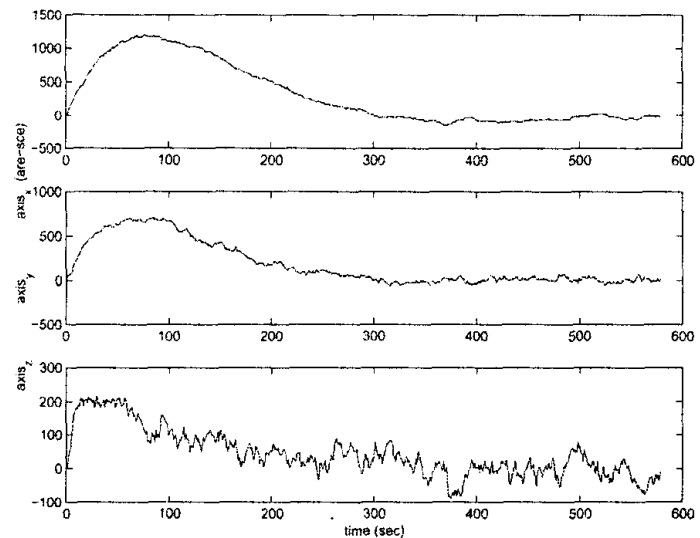

Figure 4: Attitude errors in simulation with initial constant gyro bias errors
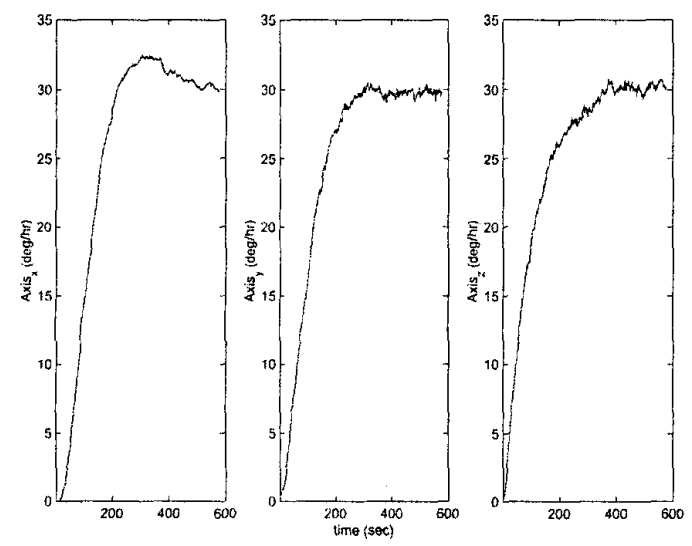

Figure 5: Estimated gro drift biases

\section{Conclusion}

In this paper, we propose a constant Kalman gain method for filter design in attitude determination system using GPS and gyros measurements. The design procedures are listed below:

1.Analyzing noise PSDs of GPS and gyros measured errors.

2.According to PSDs, determine the cut-off frequencies.
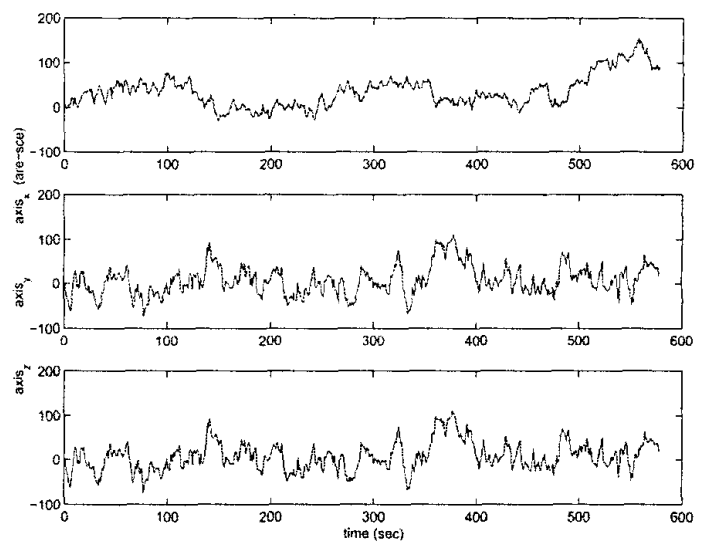

Figure 6: Attitude errors in simulation after the estimation of gyro bias errors being convergence

3.Calculating parameter $P_{a}$ (equation (39)).

4. Selecting convergence time of drift bias estimator, $T_{d}$, and computing $P_{d}$.

5.Computing Kalman $K$ (equation (24)).

\section{References}

[1] FARRENKOPF, R. L.: 'Analytic steady-state accuracy solutions for two common spacecraft attitude estimators', Journal of Guidance, Control, and Dynamics, 1978,1 (4) pp. 282-284

[2] IEEE Aerospace and Electronic Systems Society: 'IEEE Standard Specification Format Guide and Test Procedure for Single-Axis Laser Gyros', IEEE st.d $647-1995$

[3] JAY, F. A. and MATTEW, B.: 'The Global Positioning System and inertial navigation' (McGrawHill, New York, 1998)

[4] PARK C.: 'Comments on "Relationships Between Dilution of Precision for Point Positioning and for Relative Positioning with GPS', IEEE Transactions on Aerospace and Electronic Systems, 2000, 36 (1) pp. $315-316$

[5] PENG, H. M., CHANG, F. R. and WANG, L. S.: 'Rotation method for direction finding via GPS carrier phases', IEEE Transactions on Aerospace and Electronic Systems, 2000, 36 (1) pp. 72-84

[6] WERTZ, J. R.: 'Spacecraft attitude determination and control' (Kluwer Academic Publishers, 1978.) 